\section{SUSPECTED eosinophilic pustular folliculitis presenting as rapidly evolving acneiform eruption}

Editor

A 48-year-old African man presented to our Department of Dermatology, complaining of itchy papules and pustules rapidly spreading to cover the entire face (Fig. 1a and b). Dermatological assessment revealed several sterile follicular itchy papulo-pustules arranged in annular clusters and superimposed in plaques, with a tendency for central clearing and peripheral expansion, on an erythematous lacquered base mainly located on the face. Lesions healed without scarring leaving hyperpigmentation. There were no signs of lymphadenopathy, hepatosplenomegaly or petechiae. Preliminary laboratory tests demonstrated a mild leucocytosis $\left(12,4 \times 10^{9}\right.$ per L) and mild eosinophilia $(804 / \mu \mathrm{L})$; however, no haematological abnormalities were present on the peripheral smear. Serological testing for HIV, hepatitis B and C was negative. The patient was stable and immunocompetent. (a)

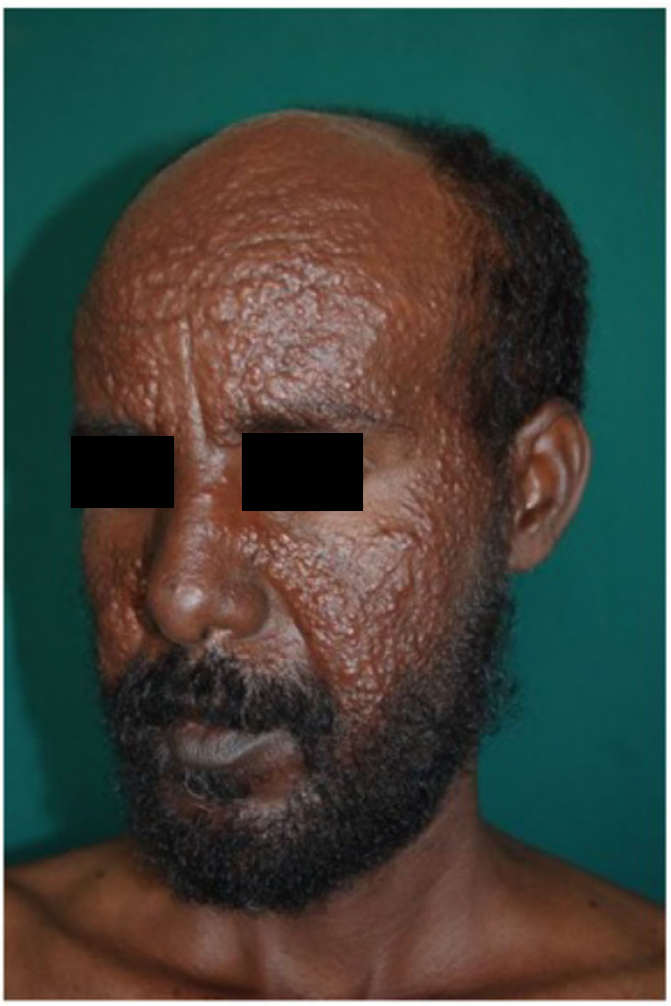

(b)

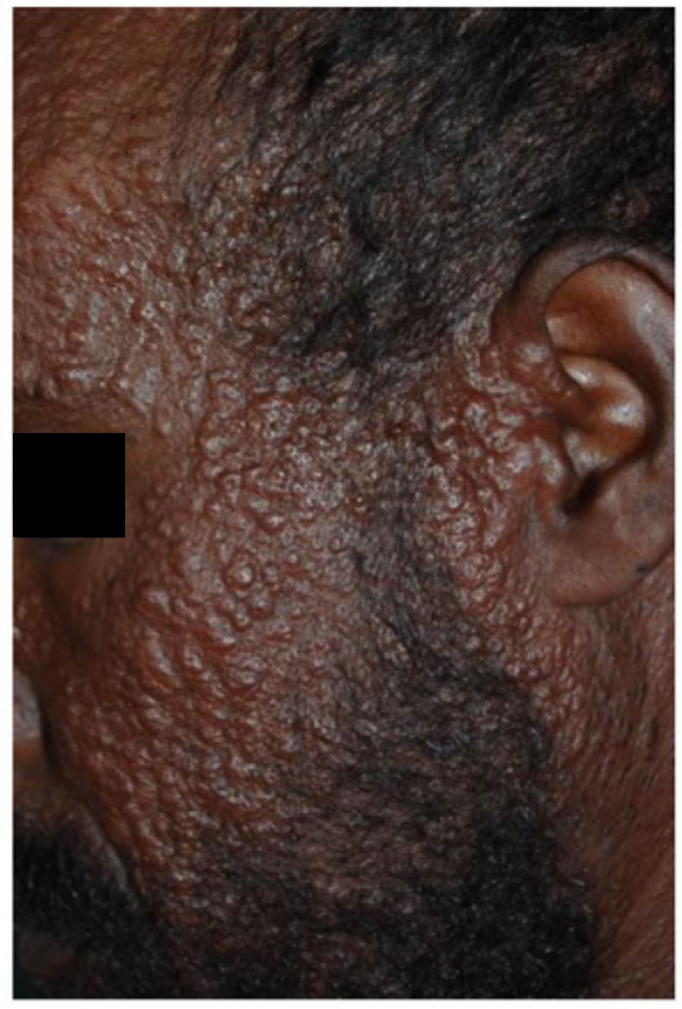

Figure 1 Papulopustular eruption limited to the face (a). The follicular lesions present an annular pattern with the tendency of central resolution (b). 
Histopathologic examination of a non-excoriated fresh papule revealed several mature eosinophils infiltrating the pilosebaceous unit with scattered neutrophils and monocytes. A diagnosis of eosinophilic pustular folliculitis (EPF) or papuloerythroderma (PEO) of Ofuji, classical type was established. Rapid improvement of lesions was noted within four weeks of systemic indometacin $75 \mathrm{mg} /$ day combined with narrow band UVB (NB UVB). NB UVB phototherapy was administered thrice weekly on non-consecutive days, and the initial dose corresponded to $70 \%$ of the NB UVB MED. No recurrences were noted after 1 year of follow-up. Classic EPF is a rare dermatosis first described in Japanese population, characterized by coalescence of brownish papules developing the typical erythroderma not involving the skin folds and giving rise to the so-called deck-chair sign. Pruritus, lymphopenia and eosinophilia are commonly associated. ${ }^{1}$ The aetiology of this disorder is still unknown but an association with acute lymphatic leukaemia, B and $\mathrm{T}$ lymphomas as well as visceral tumours have been reported. ${ }^{2,3}$ Reports of EPF occurring in Africans are even less common. The classical form affects immunocompetent patients and starts with papulo-pustules on the face rarely spreading to trunk. Immunosuppressed patients develop erythematous annular raised large follicular itchy pustules. The differential diagnosis includes conditions from banal acne to follicular mycosis fungoides. ${ }^{4}$ Several treatments have been proposed including topical/systemic steroids, PUVA, UVB, retinoids, cyclosporine and interferon alpha with different results. ${ }^{5}$ Considering the side-effects of PUVA and also the positive results obtained in the first report attesting the effectiveness of UVB in EPF in six patients with acquired immunodeficiency syndrome, ${ }^{6}$ we decided to treat our immunocompetent patient with $\mathrm{NB}$ UVB.
Our case suggests that despite its rarity, EPF should be taken into account in case of a recurrent pustular itchy rash and that phototherapy with NB UVB could be a successfully and safe treatment option.

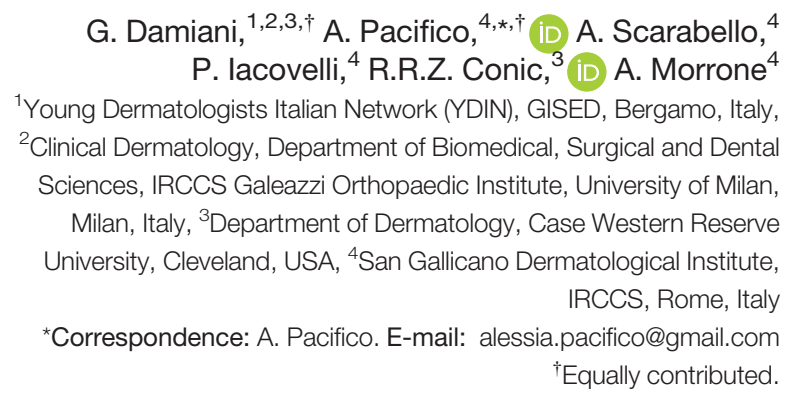

\section{References}

1 Aste N, Fumo G, Conti B, Biggio P. Ofuji papuloerythroderma. J Eur Acad Dermatol 2000; 14: 55-57.

2 Wong CJ, Houghton JB, Andrew S, Griffiths CEM. Papuloerythroderma of Ofuji associated with acute myeloid leukaemia. Clin Exp Dermatol 2003; 28: $277-279$.

3 Nomura T, Kodama K, Moriuchi R et al. Papuloerythroderma of ofuji associated with early gastric cancer. Int J Dermatol 2008; 47: 590-591.

4 Martinez-Barranca ML, Munoz-Perez MA, Morales-Garcia I, Fernandez-Crehuet JL, Sefura J, Camacho F. Ofuji papuloerythroderma evolving to cutaneous T-cell lymphoma. J Eur Acad Dermatol Venereol 2005; 19: 104-106.

5 Mutluer S, Yerebakan O, Alpsoy E, Ciftcioglu MA, Yilmaz E. Treatment of papuloerythroderma of Ofuji with Re-PUVA: a case report and review of the therapy. J Eur Acad Dermatol Venereol 2004; 18: 480-483.

6 Buchness MR, Lim HW, Hatcher VA, Sanchez M, Soter NA. Eosinophilic pustular folliculitis in the acquired immunodeficiency syndrome. Treatment with ultraviolet B phototherapy. N Engl J Med 1988; 318: 1183-1186.

DOI: $10.1111 / j d v .15893$ 